\title{
ARTICLE
}

\section{Photophysical Investigation of 8-amino 2-Naphthol in Different Micellar Environment}

Received 01th October 2019 Accepted 01th October 2019 DOI: $10.1039 / \times 0 \times x 00000 x$

\author{
Neetu Pandey ${ }^{a}$, Richa Gahlaut ${ }^{a}$, Pramod Pandey ${ }^{b, c, d}+$, Priyanka Arora ${ }^{a}$, Kalpana Tiwari ${ }^{a}$, Neeraj K. \\ Joshi $^{\mathrm{a}}$, Neeraj Tiwari ${ }^{\mathrm{a}}$, Hem C. Joshi ${ }^{\mathrm{e} *}$ and Sanjay Pant ${ }^{\mathrm{a} *}$
}

\section{Introduction}

Ability of surfactants to solubilize the membrane proteins is extremely important in simulating the complex environmental condition present in larger bio aggregates such as biological membranes [1] Surfactants are molecules containing hydrophobic long-chain with a polar head group. The head group may be ionic or neutral. It is well-known that many surfactant molecules form aggregates when their concentration exceeds a certain critical value. The aggregates, formed in water or other highly polar protic solvents, with the polar head groups pointing outwards are called micelles [2].

Research on micellar media is very important due to their micro environmental similarity to proteins,

\footnotetext{
a. Photophysics Laboratory, Department of Physics, DSB Campus, Kumaun

University, Nainital-263002, India.

b. Chemical Laboratory, Department of Chemistry, DSB Campus, Kumaun University, Nainital-263002, India.

Dalian Institute of Chemical Physics, Chinese Academy of Sciences, 457

Zhongshan Road, Dalian 116023, China

d. Department of Applied Sciences, BT Kumaun Institute of Technology, Dwarahat

Uttarakhand, India

e. Institute for Plasma Research, Laser Diagnostics Division, Bhat, Near Indira Bridge, Gandhinagar, Gujarat 382428, India

+ Current address.

*Corresponding author

Tel.: +91 7923962077 (H. C. Joshi), +91 5942237450 (Sanjay Pant).

neetupandey2006@gmail.com (N.Pandey), hem sup@yahoo.co.uk (H.C. Joshi), sanjaypent@yahoo.com (S. Pant).

Electronic Supplementary Information (ESI) available:

See DOI: $10.1039 / x 0 x x 00000 x$
}

enzymes etc., their bio-mimicking nature and capability of some of them to efficiently accommodate and transport drugs [3-7]. The most significant property of an organized assembly of nano dimensions is its ability to stabilize and bind solute molecules that are typically insoluble or sparingly soluble in a pure bulk solvent. Upon binding, a molecule experiences different environment inside the micro heterogeneous structure of micelle as compared to that in the bulk solvents. Properties like polarity, steric rigidity, viscosity and diffusion of water molecules towards the core of the micelle are different from the bulk phase [8].

Micelles have been used as models for deciphering the effect of micro heterogeneous environments on reaction dynamics and mechanisms [9-12]. Micelles are characterized by two regions- a hydrophobic core and a hydrophilic surface that may be cationic, anionic and non-ionic. All the three cationic, anionic, non -ionic type of micelles consist of a dry hydrocarbon core surrounded by a wet spherical shell called the Stern layer with thickness 6-10 for ionic micelles such as anionic Sodiumdodecylsulphate (SDS) and cationic Hexadecyltrimethylammonium Bromide (HTAB) and $25 \AA$ of palisade layer of non-ionic micelle TX- 
100. For SDS and $H T A B$, the Stern layer contains the counter ions and a considerable amount of water. The Stern layer is surrounded by a diffuse layer called the Gouy Chapman layer. This outer layer is about several hundred angstroms wide [7]. The ionic micelle consists of a micelle-water interface and the charge of this interface can such as non-ionic TX-100, cationic HTAB and anionic SDS and their micelles formation in modulated by the embodiment of sensor molecules or ion [13-17]. Structures of non-ionic surfactant TX-100, ionic HTAB and SDS and micelle formation are shown in scheme 1

Photophysical study of a probe confined in such micellar systems has been the subject of intense research in recent years revealing the microenvironment of micelles vice versa, heterogeneity and micro environmental effects of micelles on excited state dynamics have been noticed [18-22]. Naphthol and its derivatives have been of considerable interest because of their phototropic behavior in homogeneous and heterogeneous environments. Excited state behavior of naphthol and its derivatives have shown great sensitivity towards surrounding media [23-30]. Substitution of electron withdrawing group at different positions results in remarkable changes in its prototopic behaviours [31-34].The case of electron donating amino ($\mathrm{NH} 2$ ) group present at different positions has been studied theoretically as well as experimentally under various $\mathrm{pH}$ conditions [35-37].

Theoretical predication by Rayyes and Hunt et al. revealed that water plays an important role in intramolecular transfer of proton in 8-amino-1naphthol by connecting via a bridge between amino and hydroxyl groups. They postulated that hydroxyl proton is transferred to water followed by from water to amino group[31]. Similar results were also obtained by Gahlaut et al. in 8-amino 2napthol (8A2NP) in water with formation of zwitterions in the excited state [37]. In view of this it is very important to know, how the prototopic behavior changes with the amount of water in the vicinity of this molecule. Hence it would be remarkable to study this probe in the micellar environment.
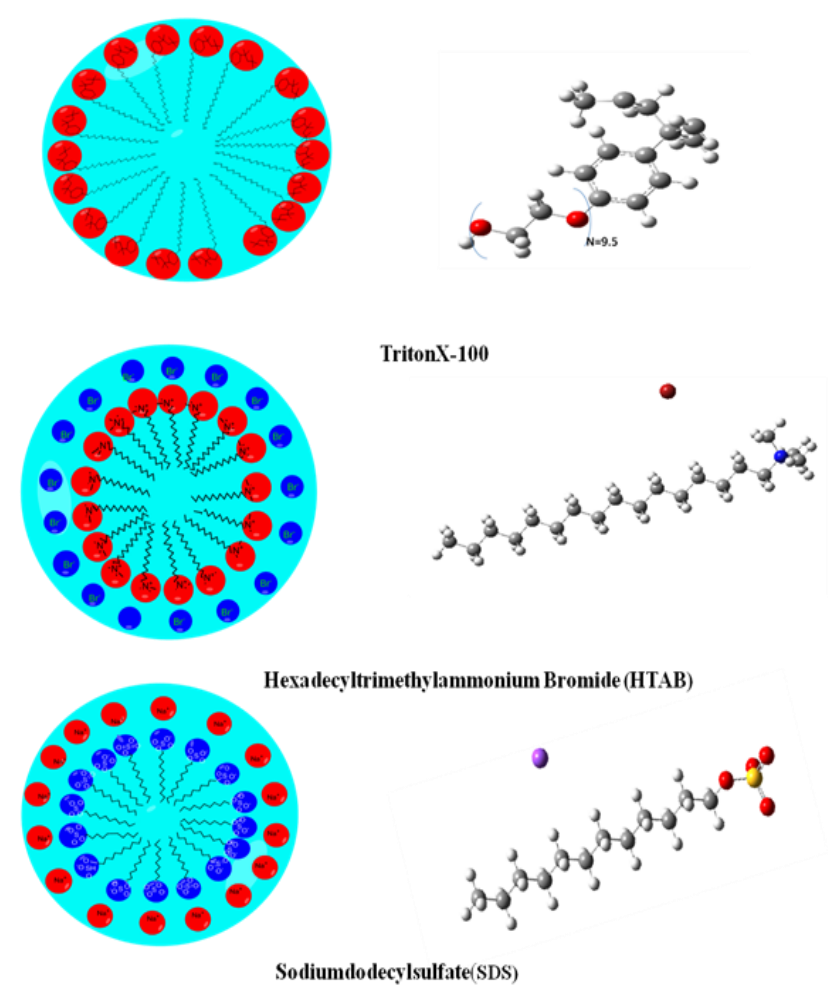

Scheme1: Formation of micelle in different surfactant and its monomer structure.

Moreover, $8 \mathrm{~A} 2 \mathrm{NP}$ is an interesting molecule containing both proton acceptor $(-\mathrm{NH} 2)$ and proton donating $(-\mathrm{OH})$ groups. Our recent investigation on the photophysics / photochemistry of $8 \mathrm{~A} 2 \mathrm{NP}$ reveals that its prototropic behavior is sensitive to the solvent polarity and $\mathrm{pH}$. Interesting photophysical properties of 8A2NP in homogeneous environment prompted us to study the variation on its prototopic behavior in different surfactants. In the present work, 8A2NP has been studied in cationic (CTAB), anionic (SDS) and non-ionic (TX-100) surfactant and changes in the excited state deactivation behavior in these surfactants are explored.

\section{Experimental}

\subsection{Materials}

8A2NP was obtained from Aldrich and is used as such. All the solvents used were either of 
spectroscopic grades or were checked for their fluorescence purity. Single distilled water $(\mathrm{pH}=6)$ was used in these experiments. The samples were prepared by dissolving appropriate concentration of 8A2NP. The surfactants SDS, HTAB and TX-100 were purchased from Sigma Aldrich and used as supplied.

\subsection{Instrumentation}

Steady state absorption spectra, were recorded by a dual beam JASCO V-550 spectrophotometer at room temperature. The excitation and emission spectra were recorded by using a JASCO FP- 777 spectrofluorimeter. The samples were excited in frontal geometry to rule out the inner filter effect. Fluorescence decay times were recorded with the help of an Edinburgh - 199 time domain spectrometer and analysed by TCC -900 software. The excitation source was a thyratron-gated hydrogen filled nanosecond flash lamp. Lamp profile was measured at the excitation wavelength using a Ludox scatterer. The pulse width was about 1.7 ns with repetition rate of $30 \mathrm{kHz}$. Time correlated single photon counting (TCSPC) technique was used to collect the decay curves and the resolution of the system was about 200 ps. The number of counts in the peak channel was at least 10,000. Time-resolved fluorescence decay curves were analyzed by deconvoluting the observed decay with the instrument response function (IRF) to obtain the intensity decay function represented as a sum of discrete exponentials

$$
I(\tau, t)=\sum_{i} \alpha_{i} e^{-t / \tau_{i}}
$$

where I (t) is the fluorescence intensity at time $t$ and $\alpha \_i$ is the amplitude of the $i^{\text {th }}$ life time such that $\sum_{-} \mathrm{i} \alpha_{-} \mathrm{i}=1$.

Quantum yields (Q.Y., $\phi$ ) were determined using quinine sulfate in $0.5 \mathrm{M} \mathrm{H} 2 \mathrm{SO} 4$ ( $\phi r e f=0.55$ ) as the reference and using the following relation:

$$
\Phi=\Phi_{\text {ref }} \frac{A_{\text {ref }} I_{5} n_{s}^{2}}{A_{5} I_{\text {ref }} n_{\text {ref }}^{2}}
$$

Where $I_{s}$ and $I_{\text {ref }}$ are the integrated fluorescence intensities, $A_{s}$ and $A_{\text {ref }}$ are the absorbance and $n \_s^{\wedge} 2$ and $[n \rrbracket$ ref^ 2 are the refractive indices of the sample and reference respectively.

\section{Results and discussion}

\subsection{Fluorescence studies of 8A2NP in water ( $\mathrm{pH}=6)$}

Aqueous solution of 8A2NP shows absorption and intense emission maxima at $337 \mathrm{~nm}$ and $448 \mathrm{~nm}$, respectively. It has been reported [37] that zwitterion is the most probable emitting species of $8 \mathrm{~A} 2 \mathrm{NP}$ in water. In analogy to the previous report neutral form of 8A2NP exists in ground state, and zwitterions in the excited state are responsible for the emission in water.
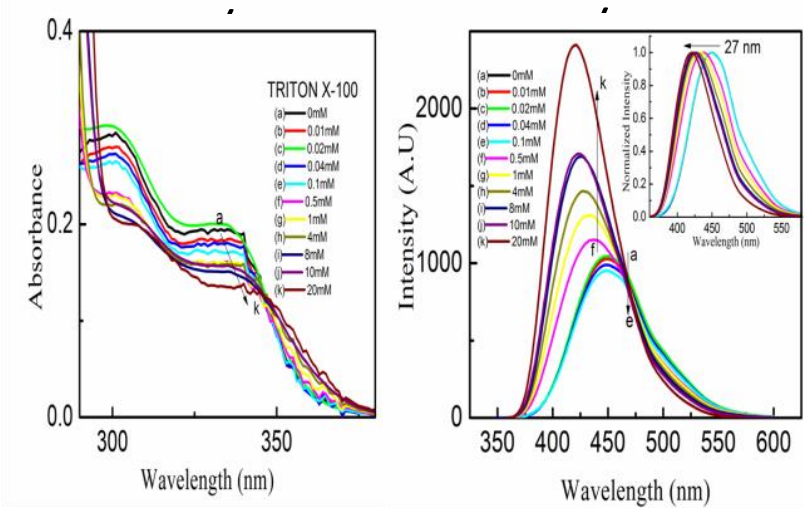

Fig 1: Absorption and emission spectra of 8A2NP in aqueous solution on addition of TX-100.

In 8A2NP, zwitterion formation cannot occur easily as proton accepter amino group(- $\left.\mathrm{NH}_{2}\right)$ is not close enough to proton donor $(-\mathrm{OH})$ group. In that case, water makes the wire network to facilitate the hydrogen atom transfer/ proton transfer (HT/PT) reaction between amino and hydroxyl group resulting in zwitterions. Formation of zwitterions in the excited state is presented in scheme 2. 


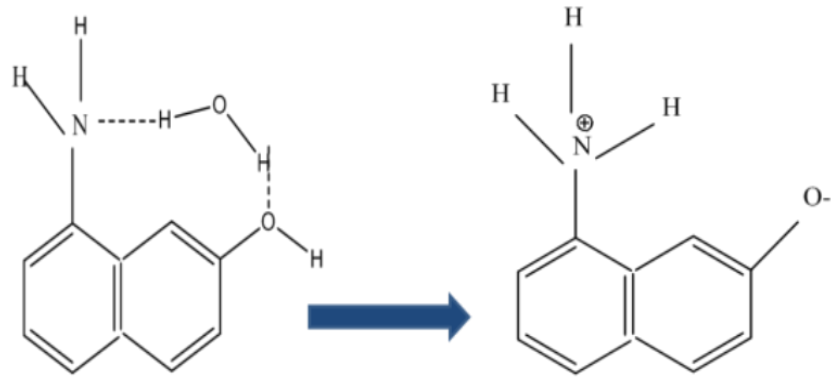

Scheme.2 : Formation of zwitterions of 8A2NP in Water

\subsection{Fluorescence studies of 8A2NP in surfactants (i) Non lonic Surfactant (TritonX -100)}

In neat aqueous solution ( $\mathrm{pH}^{\sim 6.0)}$, 8A2NP exhibits absorption maximum (S0]S1band) at $~ 337 \mathrm{~nm}$. Keeping the solute concentration fixed and upon adding TX -100 in aqueous solution, absorption maximum remains unaltered below $0.30 \mathrm{mM}$. However, a slight red shift is observed in absorption peak (Fig. 1(a)) above this concentration and it shifts to $343 \mathrm{~nm}$ at $20 \mathrm{mM}$ concentration of TX-100. Optical density decreases continuously with addition of TX-100 in the aqueous solution of 8A2NP. Red shift in the absorption maximum for higher concentration of TX-100 as compared to water is identical to that observed in alcohols [37] (;red shift in alcohol was noticed when compared with water ). It appears that the probe resides in the region where it experiences less polarity in non-ionic micelles. As reported, $\mathrm{pH} /$ polarity in micellar media is different to the bulk solvent [38].

On addition of TX-100, a small drop in the emission intensity is noticed below $0.30 \mathrm{mM}$ concentration of TX-100, however, shape of the emission profile remains unaltered. Above this concentration, emission intensity get enhanced with blue shift in the emission maximum.(Fig. 1(b)). The emission maximum shifts from $448 \mathrm{~nm}(\mathrm{TX}-100=0 \mathrm{mM})$ to $421 \mathrm{~nm}(T X-100=20 \mathrm{mM})$. The ratio of the fluorescence intensity to initial intensity (TX-100 = $0 \mathrm{mM}$ ) and quantum yield (Q.Y.) with various TX100 concentrations is shown in figures 2(a) and 2(b) respectively. It is observed from Fig. 2(b) that Q.Y. decreases up to concentration $0.30 \mathrm{mM}$, and above this concentration, enhancement in
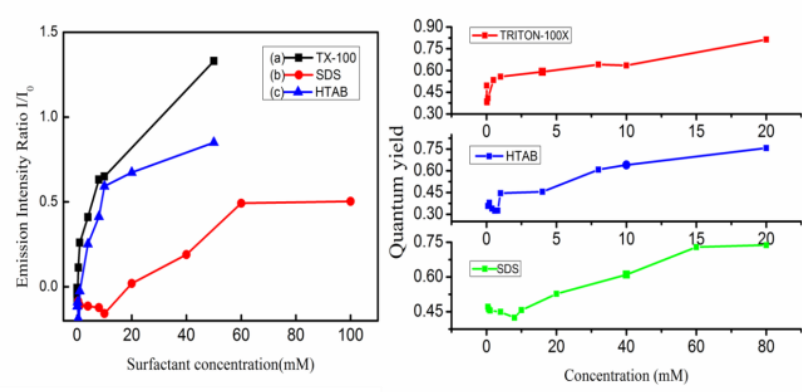

Figure 2(a) and 2(b): Variation in fluorescence intensity ratio $\left(I / I_{0}\right)$ of $8 A 2 N P$ as a function of surfactant concentration and variation in Quantum Yield of 8A2NP with varying concentration of surfactants.

fluorescence intensity has been observed. Effect of TX-100 concentrations on the excitation spectra is shown (supplementary information fig. 1) with emission monitored at $450 \mathrm{~nm}$. Shift in the excitation spectra indicates that ground state species have different environment in neat solvent as compared to the micelle environment. As we have already mentioned that this may be due to the fact that molecule resides in different polarity region.

(ii) In Cationic Micelles (HTAB):

With increasing concentration of HTAB in aqueous solution of $8 \mathrm{~A} 2 \mathrm{NP}$, absorption and emission spectra are shown in fig 3(a) and fig 3(b), respectively. The absorption characteristics such as absorption maximum and absorbance do not change below $0.80 \mathrm{mM}$ of HTAB. However, above this concentration absorption maximum gets red shifted (337 nm - $347 \mathrm{~nm}$ ) at $20 \mathrm{mM}$ concentration of HTAB. The absorption spectrum of 8A2NP in HTAB is similar to as observed in the basic medium, indicates the existence of anionic form in cationic micelles [37].

Emission intensity decreases slightly below 0.80 mM HTAB, which represents the onset of micellar aggregation. Above $0.80 \mathrm{mM}$ concentration of $H T A B$, enhancement in the fluorescence intensity with blue shifted emission (448 nm - $423 \mathrm{~nm}$ ) is observed in the emission spectra (fig. 4(b)). Fluorescence quantum yield also increases from 
0.49 (in water) to 0.78 (table 1 and fig 2(b)) at 20 $\mathrm{mM}$ concentration of HTAB. Excitation spectra (a)

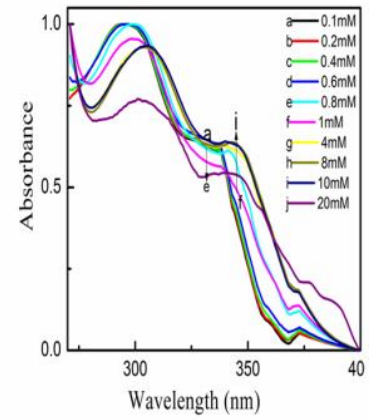

(b)

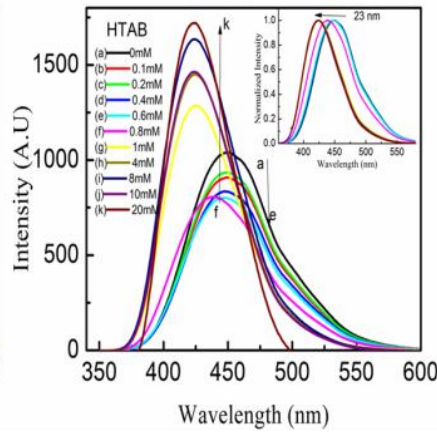

Figure 3(a) and 3(b): Absorption and emission spectra of 8A2NP with varying the concentration of HTAB.

(supplementary information fig. 1) show red shift on increasing the concentration of HTAB.

\section{(iii) In Anionic Micelles (SDS)}

In 8A2NP, on addition of anionic micelles SDS, there is no observable change in absorption spectra. The Absorption spectra (fig 4(a)) clearly reveal that in SDS micelles, unlike TX-100 and HTAB, no shift in absorption maximum is present even at very high concentration of SDS. Excitation spectra in SDS are also the replica of the absorption spectra. These results indicate that molecule does not bind well in the ground state with the anionic SDS and resides near the water surface or even in the bulk water.

The change in fluorescence intensity on varying the concentration of SDS is depicted in figure (4(b)). Below a particular concentration $\sim 8 \mathrm{mM}$ of SDS, intensity is decreased with no shift in emission maximum has been observed. However, above this concentration fluorescence intensity gets enhanced with blue shift in emission maximum (fig. 5(b)) . Variation in fluorescence intensity with addition of SDS to initial intensity $(1 / 10)$ is shown in figure $4(\mathrm{~b})$. The inset (fig.4 (b)) shows the variation of emission maxima as a function of SDS surfactant concentration. It shifts $17 \mathrm{~nm}$ from $448 \mathrm{~nm}(0 \mathrm{mM})$ to $431 \mathrm{~nm}(100 \mathrm{mM})$ in SDS. (a)

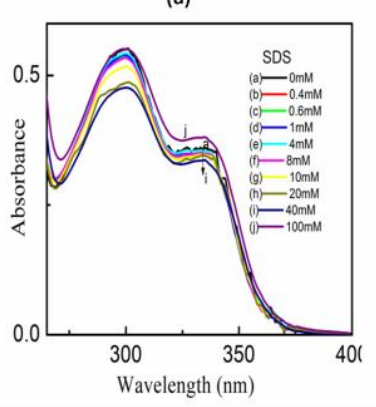

(b)

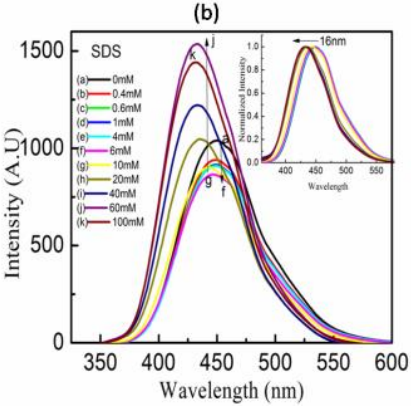

Figure 4(a) and 4(b): Absorption and emission spectra of 8A2NP with varying the concentration of SDS.

Further, the spectral and photophysical behavior of 8A2NP in the presence of surfactant can be understood by estimating some basic parameters such as binding constant, critical micellar concentration (CMC) or change in free energy of 8A2NP with different micelles.

\section{3 .3 Estimation of the Probe-Micelle Binding Constant}

The enhancement and spectral shift in the fluorescence emission of probe in micellar solution can be rationalized in terms of binding of the probe with the micelles. Binding constants of 8A2NP with different micelles are determined from the fluorescence intensity data described by Almgren et al. [39]. The binding constant [K] can be obtained by the relation:

$$
\frac{I_{\infty}-I_{0}}{I_{t}-I_{0}}=1+\frac{1}{K[M]\}}
$$

where $1 \infty, 10$ and It are the fluorescence intensities of the fluorophore under complete micellization, in the absence of micellization and in the presence of intermediate amount of surfactant respectively. [M] is related to the total concentration of the micelle and is calculated using following the equation.

$$
\mathrm{M}=\frac{\mathrm{S}-\mathrm{CMC}}{\mathrm{N}}
$$


Where S represents the surfactant concentration and $\mathrm{N}$ is the aggregation number of the micelle. The $\mathrm{N}$ values used in the calculation of [M] are 62, 60 and 143 for SDS, HTAB and TX-100 respectively [40].

A typical plot of $(|\propto-| 0) /(I t-10)$ versus $1 /[\mathrm{M}]$ for all the surfactant shows linearity (supplementary information fig 2-4). The measured $K$ values (KSDS < KCTAB < KTX-100) are given in Table 1. In non-ionic micelle, the binding constant $(K)$ is higher due to more stabilization of the probe in the micellar environment compared to the ionic micelles. Due to very thick ( $25 \AA$ ) palisade layer, most of the molecules may reside in this layer of the neutral TX-100 micelle whereas the fluorescent probe may reside in the interfacial region of SDS and HTAB as they have significantly thinner (6-10Å) Stern layer. On the other hand, binding constant of $8 A 2 N P$ with $H T A B$ is higher than SDS because the head group of SDS in the Stern layer is more hydrated than that of HTAB.

From the $K$ values, the free energy changes $(\Delta G 0=$ $-R T$ InK) for the probe-micelle binding interaction in different micelles have been calculated at $293 \mathrm{~K}$ and the corresponding values are tabulated in Table 1 . The change in the free energy associated with the probe-micelle binding process in different micellar systems shows that access of the probe in micellar phase in TX-100 is more likely than that in HTAB and SDS.

Table 1: Spectral parameter of 8A2NP in different micellar media

\begin{tabular}{llllll}
\hline Media & $\begin{array}{l}\text { Absorption } \\
\text { maxima } \\
\lambda_{\mathrm{ab}}(\mathrm{nm})\end{array}$ & $\begin{array}{l}\text { Emission } \\
\text { maxima } \\
\lambda_{\mathrm{em}}(\mathrm{nm})\end{array}$ & $\begin{array}{l}\text { Quantum } \\
\text { yield }(\Phi)\end{array}$ & $\begin{array}{l}\text { Binding } \\
\text { Constant } \\
\left(\times 10^{3}\right) \\
(\mathrm{K}) / \mathrm{M}^{-1}\end{array}$ & $\begin{array}{l}\text { Free } \\
\text { energy } \\
(-\Delta \mathrm{G}) \\
(\mathrm{kJ} \mathrm{mol}- \\
1)\end{array}$ \\
\hline Water & 337 & 448 & 0.49 & - & \\
\hline $\begin{array}{l}\text { SDS } \\
(80 \mathrm{mM})\end{array}$ & 337 & 432 & 0.73 & 0.404 & 14.14 \\
\hline $\begin{array}{l}\text { TX-100 } \\
(20 \mathrm{mM})\end{array}$ & 342 & 421 & 0.81 & 18.63 & 23.33 \\
\hline $\begin{array}{l}\text { HTAB } \\
(20 \mathrm{mM})\end{array}$ & 345 & 423 & 0.75 & 12.85 & 22.73 \\
\hline
\end{tabular}

\subsection{Determination of Critical Micelle Concentration}

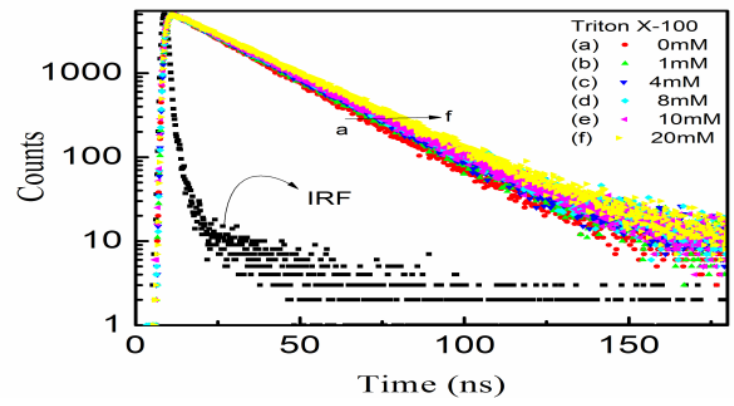

Figure 5: Decay profiles of $8 \mathrm{~A} 2 \mathrm{NP}$ as a function of concentration of Triton X-100

Surfactant molecules having a long chain form micellar aggregates above the critical micelle concentration (CMC) and hence the microenvironment around the probe molecule before and after CMC is quite different. 8A2NP is used to estimate the $\mathrm{CMC}$ values for these three surfactants and typical plots of fluorescence intensity variation with surfactant concentration are shown in supplimentry information (fig. 2-4) and the estimated CMC values are given in Table.2. It can be seen that the obtained CMC values using $8 \mathrm{~A} 2 \mathrm{NP}$ as probe are in good agreement with the reported values [5].

\section{3 .5 Fluorescence Life time measurements}

The fluorescence lifetime of a fluorophore in micellar solutions is very sensitive to the local environment around the probe and helps to understand different interactions between the probe molecule and the micelle, the location of the probe in micelles and the local viscosity and polarity of the microenvironment [41-50]. Differential degrees of solvent motion around the fluorophore or partitioning of the fluorophore in distinct regions of a confined environment of micelles gives rise to various fluorescence life time components.

Table 2: Critical micellar concentration in different surfactants

\begin{tabular}{llll} 
Media & Measured CMC & Literature CMC & Reference \\
\hline SDS & & & \\
\hline & $8.7 \mathrm{mM}$ & $7.8 \mathrm{mM}$ & 5 \\
TX-100 & $0.31 \mathrm{mM}$ & $0.26 \mathrm{mM}$ & 5 \\
\hline HTAB & $0.80 \mathrm{mM}$ & $0.92 \mathrm{mM}$ & 5 \\
\hline
\end{tabular}




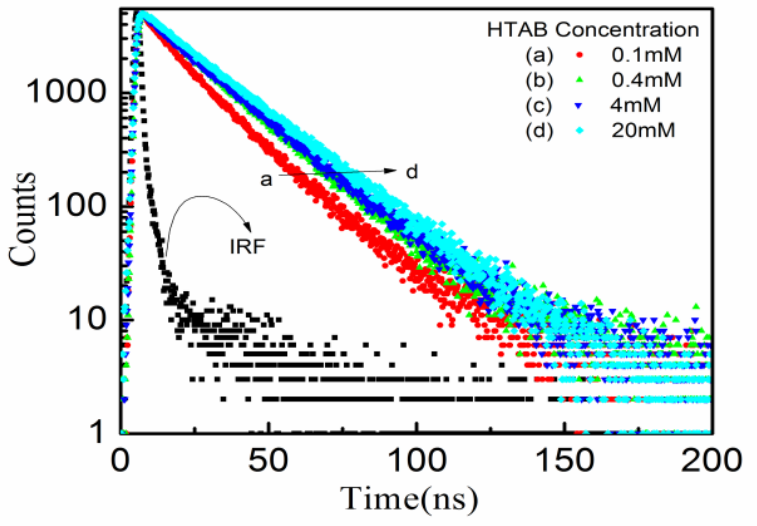

Figure 6: Decay profiles of 8A2NP with varying concentration of HTAB

In aqueous solution ( $\mathrm{pH} 6), 8 \mathrm{~A} 2 \mathrm{NP}$ exhibits single exponential decay with a fluorescence lifetime $19.52 \mathrm{~ns}$ (at $\lambda_{\mathrm{em}}=450 \mathrm{~nm}$ ), which is attributed to the decay of zwitterions.

On addition of surfactant TX-100 below CMC, mono-exponential function decays were observed.. Above CMC, decay data are fitted well with single exponential, however the decay time increases significantly from $19.87 \mathrm{~ns}(0.50 \mathrm{mM}$ of TX-100) to $22.5 \mathrm{~ns}(20 \mathrm{mM}$ of TX-100) at $450 \mathrm{~nm}$. Decay profiles of 8A2NP with varying concentration of TX-100 are shown in fig. 5.

From the steady state and lifetime data it appears that the probe resides in the palisade layer due to its larger thickness $(25 \AA)$ thereby showing large extent of blue shift and enhancement in quantum yield as well as in lifetime.

Decay analysis with varying concentration of $\mathrm{HTAB}$ is shown in table 3. Below CMC decay data are well fitted with mono-exponential function. However, above its $\mathrm{CMC}$ value, decay profile (fig.6) is found to be best fitted with a bi-exponential function with decay components of $6.5 \mathrm{~ns}\left(\alpha^{1}=5 \%\right)$ and 20 $.69 \mathrm{~ns}\left(\alpha^{2}=95 \%\right)$ at $0.1 \mathrm{mM}$ of HTAB. The shorter decay component may be attributed to anionic species and the longer one can be attributed to zwitterions. It can be noted here that the
Table 3: Fluorescence decay parameter of 8A2NP in different micellar media.

\begin{tabular}{|c|c|c|c|c|c|c|c|c|}
\hline Media & $\begin{array}{l}\text { concent } \\
\text { ration }\end{array}$ & $\begin{array}{l}\lambda_{\mathrm{em}} \\
(\mathrm{nm} \\
)\end{array}$ & $\begin{array}{l}\tau_{1} \\
\text { (ns) }\end{array}$ & $\begin{array}{l}\tau_{2} \\
\text { (ns) }\end{array}$ & $\begin{array}{l}\boldsymbol{\alpha}_{1^{*}} \\
\%\end{array}$ & $\begin{array}{l}\boldsymbol{\alpha}_{2^{*}} \\
\%\end{array}$ & $\chi_{1}^{2} \#$ & $\chi_{2}^{2} \#$ \\
\hline \multirow[t]{11}{*}{ Water } & - & 450 & 19.5 & - & - & - & 1.0 & \\
\hline & & & 2 & & & & 3 & \\
\hline & $0.1 \mathrm{mM}$ & 430 & 19.4 & & & & 1.0 & \\
\hline & & 450 & 2 & & & & 0 & \\
\hline & & & 19.7 & & & & 1.0 & \\
\hline & & & 0 & & & & 7 & \\
\hline & $0.5 \mathrm{mM}$ & 430 & 19.8 & & & & 1.1 & \\
\hline & & 450 & 2 & & & & 0 & \\
\hline & & & 19.8 & & & & 1.0 & \\
\hline & & & 8 & & & & 6 & \\
\hline & $1 \mathrm{mM}$ & 430 & 20.4 & & & & 1.0 & \\
\hline \multirow{20}{*}{ Triton } & & 450 & 0 & & & & 3 & \\
\hline & & & 20.2 & & & & 1.0 & \\
\hline & & & 5 & & & & 7 & \\
\hline & $10 \mathrm{mM}$ & 420 & 21.8 & & & & 1.0 & \\
\hline & & 450 & 8 & & & & 7 & \\
\hline & & & 21.0 & & & & 1.1 & \\
\hline & & & 9 & & & & 3 & \\
\hline & $20 \mathrm{mM}$ & 420 & 22.4 & & & & 1.0 & \\
\hline & & 450 & 9 & & & & 4 & \\
\hline & & & 22.0 & & & & 1.1 & \\
\hline & & & 8 & & & & 0 & \\
\hline & $0.1 \mathrm{mM}$ & 430 & 19.1 & & & & 1.0 & \\
\hline & & 450 & 7 & & & & 6 & \\
\hline & & & 19.3 & & & & 1.1 & \\
\hline & & & 9 & & & & 2 & \\
\hline & $0.4 \mathrm{mM}$ & 430 & 20.9 & & & & 1.0 & \\
\hline & & 450 & 7 & & & & 7 & \\
\hline & & & 20.4 & & & & 1.0 & \\
\hline & & & 1 & & & & 2 & \\
\hline & $1 \mathrm{mM}$ & 420 & 6.50 & 20.69 & 5.00 & 95.0 & 1.2 & 1.0 \\
\hline \multirow{21}{*}{ HTAB } & & 450 & 10.8 & 21.31 & 6.70 & 0 & 9 & 7 \\
\hline & & & 0 & & & 93.0 & 1.3 & 1.0 \\
\hline & & & & & & 0 & 2 & 3 \\
\hline & $10 \mathrm{mM}$ & 420 & 13.5 & 22.95 & 29.0 & 71.0 & 1.4 & 1.0 \\
\hline & & 450 & 9 & 21.38 & 0 & 0 & 0 & 5 \\
\hline & & & 11.2 & & 12.9 & 87.0 & 1.2 & 1.1 \\
\hline & & & 6 & & 5 & 5 & 6 & 6 \\
\hline & $20 \mathrm{mM}$ & 420 & 14.5 & 22.48 & 36.2 & 63.7 & 1.3 & 1.0 \\
\hline & & 450 & 9 & 21.78 & 1 & 9 & 9 & 5 \\
\hline & & & 12.2 & & 18.0 & 82.0 & 1.2 & 1.0 \\
\hline & & & 4 & & 0 & 0 & 3 & 2 \\
\hline & $0.6 \mathrm{mM}$ & 430 & 19.3 & & & & 1.0 & \\
\hline & & 450 & 2 & & & & 8 & \\
\hline & & & 19.5 & & & & 1.0 & \\
\hline & & & 0 & & & & 5 & \\
\hline & $4 \mathrm{mM}$ & 430 & 19.5 & & & & 1.0 & \\
\hline & & 420 & 2 & & & & 8 & \\
\hline & & & 19.8 & & & & 1.0 & \\
\hline & & & 5 & & & & 9 & \\
\hline & $8 \mathrm{mM}$ & 430 & 12.0 & 21 & 17 & 82 & 1.3 & 1.0 \\
\hline & & 450 & 0 & 20.53 & 8 & 92 & 2 & 6 \\
\hline \multirow[t]{9}{*}{ SDS } & & & 6.86 & & & & 1.2 & 1.0 \\
\hline & & & & & & & 4 & 4 \\
\hline & $20 \mathrm{mM}$ & 430 & 12.6 & 22.00 & 12 & 87 & 1.4 & 1.0 \\
\hline & & 450 & 2 & 22.17 & 8.8 & 91.2 & 0 & 6 \\
\hline & & & 9.14 & & & & 1.2 & 1.0 \\
\hline & & & & & & & 8 & 8 \\
\hline & $80 \mathrm{mM}$ & 430 & 14.1 & 23.04 & 9 & 90 & 1.2 & 1.0 \\
\hline & & 450 & 5 & 8 & 4.87 & 95.1 & 5 & 6 \\
\hline & & & 9.93 & 22.88 & & 3 & 1.3 & 1.1 \\
\hline
\end{tabular}


prototropic behaviour of $8 \mathrm{~A} 2 \mathrm{NP}$ for various $\mathrm{pH}$ is reported in reference [37]. In SDS, below its CMC, the decay profiles are fitted well with single exponential akin to HTAB and TX-100. However, when the concentration of surfactant exceeds the CMC, they are fitted with double exponential function. Decay profiles with varying concentration of SDS are shown in fig.11 and the fitted data are summarized in table 3 . Shorter component in decay data can be attributed to anionic species [37] and the longer component to zwitterions.

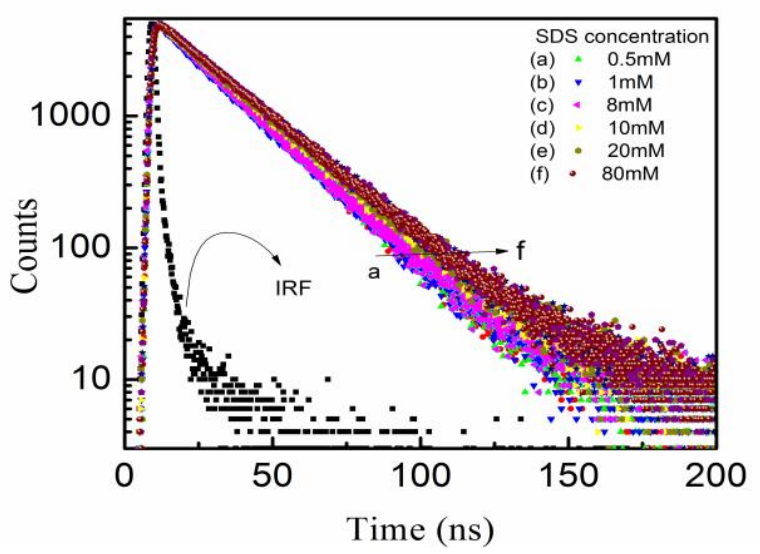

Figure 7: Decay profiles of 8A2NP with varying concentration of SDS

In short, absorption and emission properties of 8A2NP are quite sensitive to the presence of water and hence the probe bound to the micellar aggregate in water will be partially shielded with water. At concentrations well below the CMC, small premicellar aggregates may exist in the system and hence below $\mathrm{CMC}$, no considerable shift in absorption and emission peak is observed with slightly change in absorbance and emission intensity. In case of non-ionic micelles, major role is played by the hydrophobic force because of the absence of Columbic interaction. However, in ionic micelles both the hydrophobic and columbic interactions come into picture.

In case of non-ionic micelle Triton X-100, the probe gets transferred from the the polar aqueous phase to the less polar micellar region. As there is no electrostatic interaction of polar head groups in the micelle/water interface, the probe molecule can go deeper into the thicker Stern layer as is revealed by the huge binding constant. Emission maximum of 8A2NP observed in TX-100 is almost identical to the emission maximum of 8A2NP in alcohols. However, non radiative losses due to zwitterion form are decreased in micellar cage and hence enhancement in quantum yield as well as in fluorescence lifetime is observed.

In HTAB, absorption peak shows that anions are present in the ground as well as excited state which is confirmed by the decay time analysis. Further, increment in anionic contribution can be explained on the basis of greater destabilization of $8 \mathrm{~A} 2 \mathrm{NP}$ anion, due to repulsion from negatively charged hydroxyl ions of water which get accumulated in a greater concentration in the immediate vicinity of the HTAB water interface and also due to the attraction of positive head groups of HTAB micelle. Due to its inherent positive charge, HTAB is expected to have the lowest proton concentration and highest hydroxyl ion concentration in GC layer and this should facilitate the deprotonation process. Therefore most of the probe molecules reside near GC layer in HTAB as the GC layer is wider than the Stern layer. The fluorescence intensity and lifetime changes may be due to $\mathrm{pH}$ effect. As we have discussed earlier that in case of HTAB, probe molecule may experience higher $\mathrm{pH}$.

In SDS, due to the thin stern layer molecule resides in the GC layer or near to interface of stern layer which is clearly shown by low binding constant of this molecule as compared to TX-100 and HTAB. Decay analysis confirms the presence of anions in SDS. In the ground state, micelle bound with the neutrals however in excited state due to the presence of few hydroxyl ions near the surface, excited state deprotonation may occur and contribute to the anion.

The present results indicate that the zwitterions reside deeper in the micellar cage while anions in 
the interfacial region. However, larger contribution of anions in HTAB is from ground state anions present in this probe situated mainly in the interfacial region of the micelles.

\section{Conclusions}

Photophysical investigation of $\mathrm{pH}$ sensitive probe 8A2NP in heterogeneous micellar environment shows different behavior as compare to the case of bulk water. Enhancement in fluorescence intensity and blue shifted emission of 8A2NP has been observed in all the micelles. In Triton X-100, the binding constant is higher as compared to HTAB and SDS indicating that $8 \mathrm{~A} 2 \mathrm{NP}$ probably be present inside the Stern layer. Higher anionic contribution in HTAB than SDS increases its fluorescence significantly. Weak binding constant in SDS is attributed to the presence of probe molecule at the outer location. It is better understood from the present study that 8A2NP appears to be quite sensitive in probing micellar environment.

\section{Conflicts of interest}

There are no conflicts to declare.

\section{Acknowledgements}

Authors, N.Pandey and K. Tiwari are thankful to UGC, New Delhi, India for providing financial assistance in the form of research fellowships under the scheme for the meritorious students in sciences (RFSMS).

\section{References}

[1] C Tanford,The Hydrophobic effect : Formation of Micelles and Biological Membrane. Wiley: New York, 1973.

[2] (a) S. Joshi, T. Varma Y, Debi D. Pant Colloids and SurfacesA: Physicochem.Eng. Aspects 425 (2013) 59- 67 (b) D. Attwood. A T Florence, Surfactant Systems Chapman and Hall: London, 1983. (c) J. K Thomas, Chemistry of Excitation at the Interfaces; American Chemical Society: Washington, DC, 1984.

[3] T.Sakthivel, A .T Florence. Drug Deliv.Technol. 3(2003)73-78

[4] K.Anwer, C.Meaney, G. Kao, N. Hussain, R .Shelvin, R.M Earls, P .Leonard, A .Quezada, A.P Roland, S.M Sullivan, Cancer Gene Therapy 7 (2000)1156-1164

[5] V. Ramamurthy, Photochemistry in Organized and Constrained Media, VCH Publisher, New York, 1991.

[6] K. Kalyanasundaram, Photochemistry in Microheterogeneous Systems, Academic Press, Orlando, FL, 1987

[7] K.L Mittal, B .Lindman, Surfactants in solution, Plenum, New York (1994)1-3.

[8]A .Mallick, B .Halder, S .Maity, N.Chattopadhyay J Colloid Interface Sci 278 (2004) 215-223.

[9] P. Bortolus, S. Monti, Adv. Photochem. 21(1996)1.

[10] M.H.Kleinman, C.Bohne, Organic Photochemistry :Molecular and Supramolecular Photochemistry, Marcel Dekker, New York, 1997.

[11] M. Gratzel, in: M. Gratzel (Ed.), Solar Energy Harvesting, Elsevier, New York,1988, pp. 394-440.

[12] M.A. Fox, Top. Curr. Chem. 159 (1991) 67-101.

[13] G. D. J. Phillies and J. E. Yambert, Solvent and Solute Effectson Hydration and Aggregation Numbers of Triton X-100 Micelles,Langmuir, 12 (1996)3431-3436.
[14] J. V. Joshi, V. K. Aswal and P. S. Goyal, , J. Phys. Condens.Matter, 19(2007) 196219.

[15] S. S. Berr, E. Caponetti, R. R. M. Jones, J. S. Johnson and L. J. Magid,, J. Phys. Chem., 90 (1986) 5766-5770.

[16] S. S. Berr, , J. Phys. Chem., 91 (1987)4760-4765.

[17] H. H. Paradies, J. Phys. Chem., 84 (1980) 599-607.

[18]S.P. Moulik, Curr. Sci. 71 (1996) 368-375.

[19] N. Chattopadhyay, R. Dutta, M. Chowdhury, ,J.Photochem.Photobiol. A 47 (1989) 249-257. [20] A. Douhal, T. Fiebig, M. Chachisvilis and A. H. Zewail, J. Phys. Chem. A, 102(1998) 1657-1660.

[21] S. Vajda, R. Jimenez, S. J. Rosenthal, V. Fidler, G. R. Fleming andE. J. Castener Jr., , J. Chem. Soc.,Faraday Trans., 91 (1995)867-873.

[22] N. Nandi and B. Bagchi, ,J.Phys.Chem., 100 (1996)1391413919.

[23] A.A. El-Rayyes, H.P. Perzanowski, S.A.I. Barri, U.K.A. Klein, J. Phys. Chem. A 105

(2001) 10169-10175.171

[24] A. Weller, Prog. React. Kinet. 1 (1961) 189-214.

[25] W.R. Laws, L. Brand, J. Phys. Chem. 83 (1979) 795-802.

[26] E. Van der Donckt, Prog. React. Kinet. 5 (1970) 273-299.

[27] J.F. Ireland, P.A.H. Wyatt, Adv. Phys. Org. Chem. 12 (1976) 132-215.

[28] D. Huppert, M. Gutman, J.K. Kaufman, Adv. Chem. Phys. 47 (1981) 643-679.

[29] H. Shizuka, Acc. Chem. Res. 18 (1985) 141-147.

[30] N. Chattopadhyay, R. Dutta, M. Chowdhury, Ind. J. Chem. A 31 (1992) 512-517.

[31] L.M. Tolbert, K.M. Solntsev, Acc. Chem. Res. 35 (2002) 1927.

[32] K.M. Solntsev, D. Huppert, N. Agmon, L.M. Tolbert, J. Phys. Chem. A 104 (2000) 4658-4669.

[33] E-Ann. Gould, A.V. Popov, L.M. Tolbert, I. Presiado, Y. Erez, D. Huppert, K.M.Solntsev, Phys. Chem. Chem. Phys. 14 (2012) 8964-8973

[34] A.A. El-Rayyes, T. Htun, J. Mol. Struct. (Theochem) 681 (2004) 9-13.

[35] D.W. Ellis, L.B. Rogers, Spectrochim. Acta 20 (1964) 17091720.

[36] D.W. Ellis, L.B. Rogers, Spectrochim. Acta 18 (1962) 265270.

[37] R. Gahlaut, H. C. Joshi, N. K. Joshi, N. Pandey, S. Pant,SpectrochimicaActa A: Molecular and Biomolecular Spectroscopy 109 (2013) 164-172

[38]N. Sarkar, A. Datta, S. Das, K. Bhattacharyya J. Phys. Chem. 100(1996) 15483-15486

[39]. Almgren M, Griesser F, Thomas J. K . J Am ChemSoc 101 (19790279-291

[40]. G.Saroja, B.Ramachandan, S .Saha, A .Samanta J. Phys. Chem. B 103(1999)2906-2911

[41] J.R. Lakowicz,Principles of Fluorescence Spectroscopy, Second edition, Plenum, New York, 1999.

[42] A. Mishra, G.B. Behera,M.M.G.Krishna, N.Periasamy, , J. Lumin. 9 (2001)175-188.

[43] T.S.Singh,S.Mitra, , J. Colloid Interface Sci.311(2007)128134.

[44] A.Sarkar, S.Pramanik, P.Banerjee, S.C.Bhattacharya, Colloids Surf. A: Physicochem. Eng. Aspects 317 (2008) 585-591.

[45] A.Chakrabarty,A.Mallick,B.Haldar, P. Purkayastha, P. Das, N. Chattopadhyay, Langmuir 23 (2007) 4842-4848.

[46]E.R. Macedo, L.D.Boni,L.Misoguti,C.R.Mendonca, H.P.de Oliveira, , Colloids Surf. A: Physicochem. Eng. Aspects 392 (2011) 76-82.

[47]

A.Mahata,D.Sarkar,D.Bose,D.Ghosh,P.Das,N. Chattopadhyay, J. Colloid Interface Sci.335 (2009) 234-241. 
[48] S.Dhar,D.K.Rana,S.C. Bhattacharya, ColloidsSurf A: Physicochem.Eng. Aspects 402(2012) 117-126.

[49] A.Sarkar, N. Kedia, P.Purkayastha,S. Bagchi, J. Lumin. 131 (2011) 1731-1738.

[50] P.Banerjee, S. Pramanik, A. Sarkar, S.C. Bhattacharya, , J. Phys.Chem. B 112 (2008) 7211-7219.

\section{Highlights}


- In the present work, steady state and time domain spectroscopic excited state relaxation processes in amino derivative of naphthol (8-amino-2-naphthol) have been explored in different micellar environments.

- Physico-chemical properties e.g. binding constant, free energy change and critical micellar concentration of different micelles with the probe molecule are estimated.

- Variation in spectral properties of probe inside micellar cage indicates that it experiences different environment inside micelles as compared to the bulk aqueous phase.

\section{Graphical Abstract}

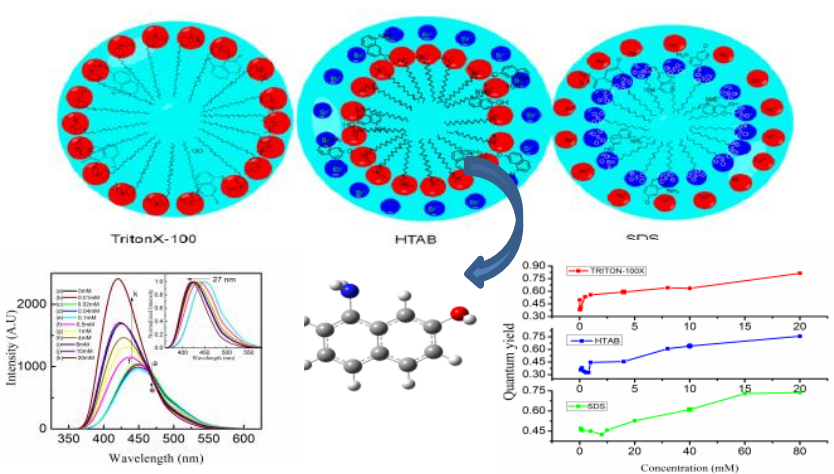

\section{Supplementary Information}

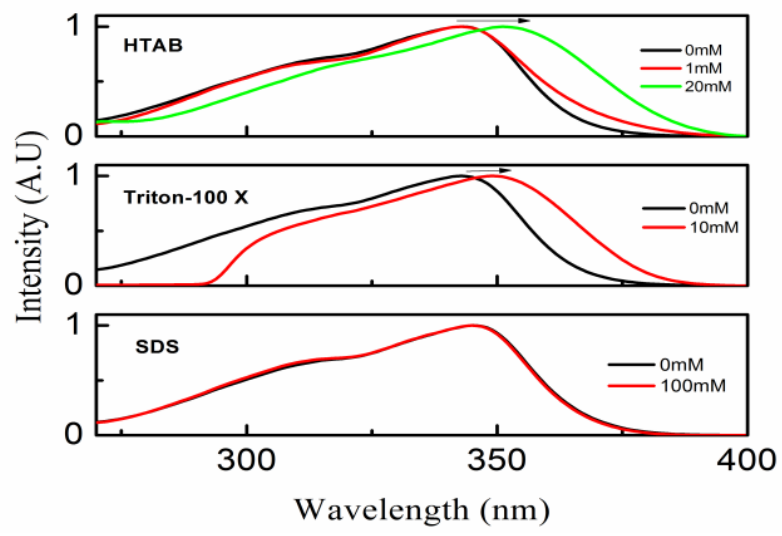

Figure1: excitation spectra of 8a2NP in surfactants for various concentrations.

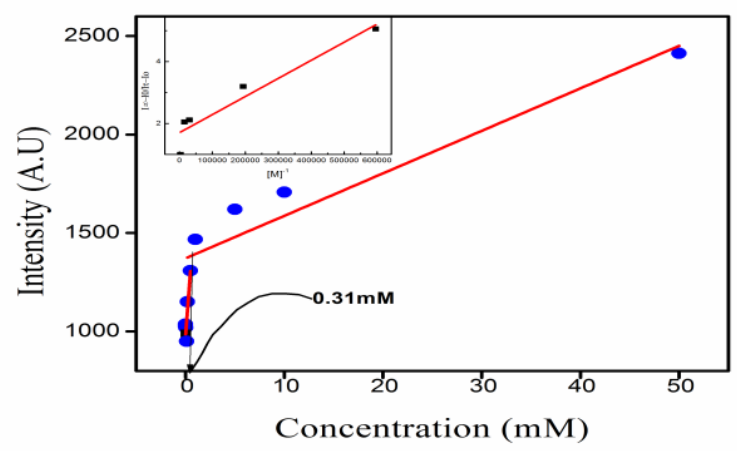

Figure 2: Variation in fluorescence intensity of 8A2NP with T X-100, Inset: Plot between $\left(I_{\infty}-I_{0}\right) /\left(I_{t}-I_{0}\right)$ verses $[M]^{-1}$

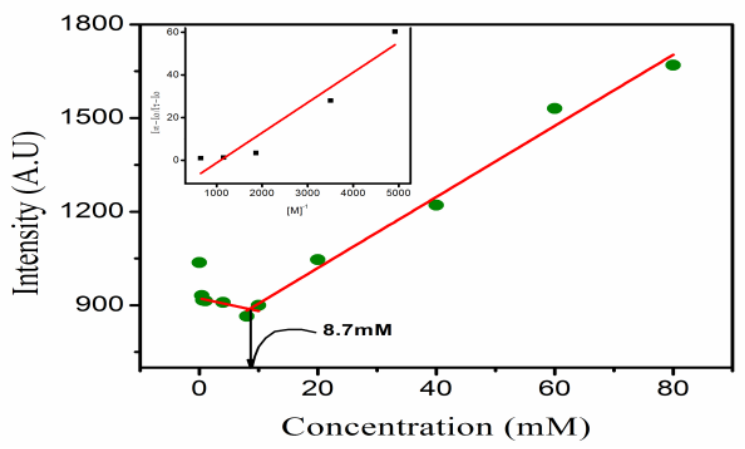

Figure 3: Variation in fluorescence intensity of 8A2NP with HTAB, Inset: Plot between $\left(I_{\infty}-I_{0}\right) /\left(I_{t}-I_{0}\right)$ verses $[M]^{-1}$ 